\title{
"BN ANATOMY" AN INTERACTIVE AUGMENTED REALITY SYSTEM FOR LEARNING BONE ANATOMY
}

\author{
João Vitor de S. Chagas, Phellipe C. Santiago, Cristina A. P. Fontes and Aura Conci \\ Universidade Federal Fluminense (UFF), Brazil
}

\begin{abstract}
The study of bone anatomy has a great impact on the courses of health courses and brings a series of challenges, since books and other 2D materials cannot present all the details of these organs. In this paper, an interactive augmented reality system for teaching this field of science is presented. The system's usability was tested by a group of volunteers, containing students and health professionals, indicating the system's ability to motivate students to learn bone anatomy.
\end{abstract}

\section{KEYWORDS}

Augmented Reality, Bone Anatomy, Gamification, Gesture Analysis, Health Games

\section{INTRODUCTION}

Health professionals are subjected to physical and psychological pressure in workplace or in academy. In this context, gamification is increasingly used as a complement to traditional teaching strategies in medical education, to increase student engagement and motivation to learning. Thinking about it, several technologies have emerged with the purpose of making the teaching process, from the most diverse branches of health, more pleasant, motivating and immersive. Applications involving augmented reality (AR) or virtual reality (VR) have a special ability to generate greater immersion in learning.

AR presents a vision of the real world that incorporates information from the virtual world to broaden the perspective of the real world (Singh and Singh, 2013). The information incorporated has a broad meaning, it can be any type of data (video, image, audio), although, in most part, this information represents images, which may be consistent or not with the real environment. To interact with the AR environment, the use of markers or gestural analyzes may be necessary. AR systems, in general, have the properties (Azuma et al., 2001) of (1) combine the real world with virtual objects within the real environment; (2) promote real-time interactivity; and (3) align real and virtual objects together.

The AR faces in its usability challenges similar to those of traditional interfaces, such as excessive information presented to the user and problems to determine the focus of the user's attention. When focused on the educational aspect, this technology comes up against the problem of content creation, since this type of project demands a great effort, with the need for computational knowledge, which creates a barrier for educators in fields other than computing to develop solutions in AR. Just as there is also a need for knowledge on the topic addressed and pedagogical, which brings obstacles for computer professionals to develop this type of solution alone that is, projects involving AR need a multidisciplinary team (Martins, 2012). In addition, it is always a challenge to combine new teaching technologies, which escape the conventional methodology, with evaluation processes that can effectively indicate the student's progress.

This article presents BN Anatomy: an application that uses AR technology for learning bone anatomy through game concepts such as: rewards, progress feedback and competition. The prototype was designed to be accessible and inexpensive since its only requirement is to install the software on a common smartphone (provided it has a rear camera and a system version superior to Android 7.0), in order to bring the experience of the bone anatomy classroom, even without the existence of real skeletons in the environment. BN Anatomy presents a great immersion for presenting a $360^{\circ}$ view of the skeleton and all its interactions happen through 3D gestural analyzes in real time, provided from the smartphone camera. 


\section{RELATED WORK}

The study of human anatomy plays an important role in doctors and nurses training. However, the practical knowledge of this area is subject to some obstacles, since in the conventional format these classes need laboratories that have dissected bodies and human skeletons to happen. Solutions involving the use of AR have already been proposed to support this study. Ferrer-Torregrosa et al. (2016) investigate didactic strategies for distance learning, based on an inverted classroom concept. This study presented an experiment with 171 students from medicine, physiotherapy and podiatry courses, divided into 3 groups. Each group received a distinct material to anatomy studies: annotations with images; videos and; an AR book. The AR group obtained a significantly higher average score and lesser dispersion on the exams than the others. Despite the promising results, the study does not address the issue of gamification, as this work does.

Andayani et al. (2019) proposed a 3D visualization system with AR for interactive medical learning of pulmonary anatomy. In this research, the authors used the Blender software for object modeling and image targeting techniques for displaying 3D objects from markers, but they did not present results regarding the usability and performance of the students, being restricted to tests of functioning of such markers.

Kurniawan et al. (2018) point out the students' difficulty in learning about anatomy of the human body through books, since 2D images cannot accurately represent the 3D body anatomy. To overcome this difficulty, researchers developed a mobile application allowing visualization of different parts of body using AR and markers. The project presents positive feedbacks; however, it only displays objects and their information, do not challenging the students in order to further increase their motivation on the topic.

Stefan et al. (2014) work is most related to this here proposed. It is a medical education and entertainment system with AR for learning bone anatomy. It is considered as an "AR bone puzzle", where Kinect is used to assemble a skeleton on users own body from the bones that are being randomly drawn, among 16 possible parts, working as a puzzle. This solution, like ours, uses gesture-based interaction, but differently of ours has a higher cost, since a Kinect is necessary (our project is based only on the use of smartphones).

\section{PROPOSED METHODOLOGY}

This section presents the purposed system based on the use of AR, gesture analysis and gamification activities to challenges and rewards students, designed to be accessible and to retain the student's attention.

\subsection{Developed Prototype}

In this prototype the APIs (Application Programming Interface) ARFoundation and ARCore were used, they provide architecture to work using AR, where it is possible to simulate the 3D model of a human skeleton in a real environment, maintaining its proportions and characteristics of virtual world. For prototype development, the Unity game development engine (version 2019.3.19f1) is also used, together with the SDK (Software Development Kit) and Devkit Manomotion that provides a structure for real-time 3D gesture analysis, requiring only a minimal computer power and a smartphone camera from for real time uses.

\subsubsection{Details Related to Bones}

We are currently working on 12 bones of the human body for interaction; these bones are represented in Figure 1. The choice of implemented bones followed the criteria of relevance to the matter, according to direct research with professionals in the area, and diversity of regions. However, more bones are being added in new versions in order to have an interactive representation of all the bones of a complete human skeleton at the end of the work.

The student should point out in the 3D model which bone is indicated in the question. Each bone has a flag that will be compared with the flag of the question drawn, so if the user hits the bone of the question another one will be drawn for a new question. The execution of the prototype can be seen in the Figure 2. 

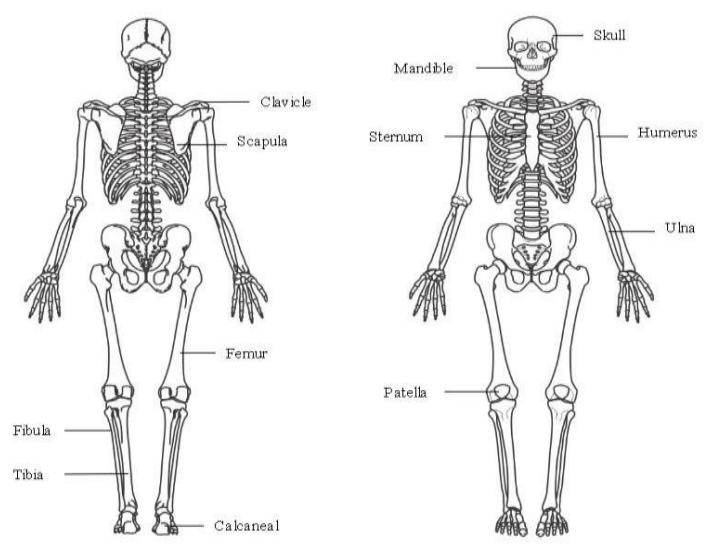

Figure 1. Skeleton images representing the interactive bones in the prototype
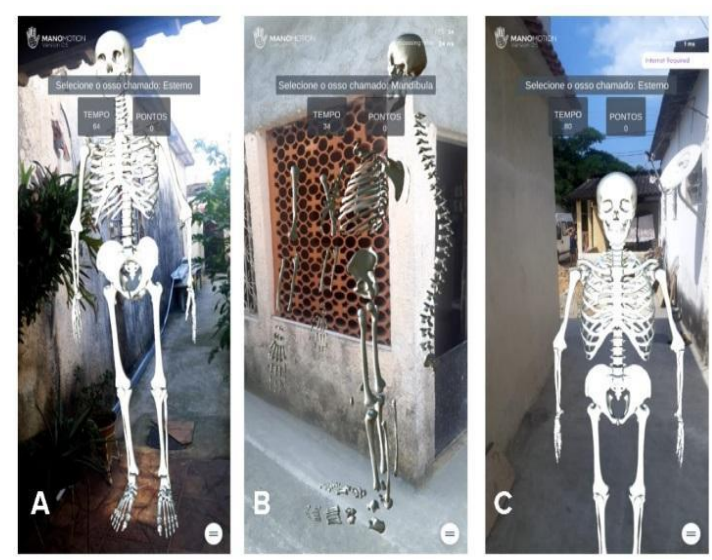

Figure 2. Virtual Skeleton projected (a) in a wooded environment (b) in a garage (c) on the street

\subsubsection{Gamification}

Quiz, scoring and time are used to improve self-learning. The player must correctly select the bone presented in questions: he receives points for hitting the bone and her points are reduced when he misses. In the punctuation system used, the skeleton was divided into 4 regions (head, chest, lower and upper limbs) the player loss more point when the answer is a bone belonging to a region other than the one presented. This system also makes use of combos, where the player gains more points for certain bones in a row. There is a time challenge of 1 minute for the user to get the highest score possible. In addition, a visualization tool allows the user to group, separates and rotates skeleton bones. These details and others can be seen well in this play: https://www.youtube.com/playlist?list=PLt-HAUhESHxVvzcVBzgt9uiTMsBkF7fT8

\subsubsection{How Augmented Reality Works in BNAnatomy and Was Tested}

Figure 3 presents a flowchart of augmented reality operation in the project. The application starts if the device meets the minimum requirements for operation, otherwise it is closed. Right after its initialization, the camera captures the environment and then detects a flat area to use as a reference when the virtual object is instantiated in the real world. If the flat area is valid, the application will calculate the position of the virtual object using the flat region of the physical world detected as a reference and the last step is to render the virtual object in the correct position within the flat region of the physical world.

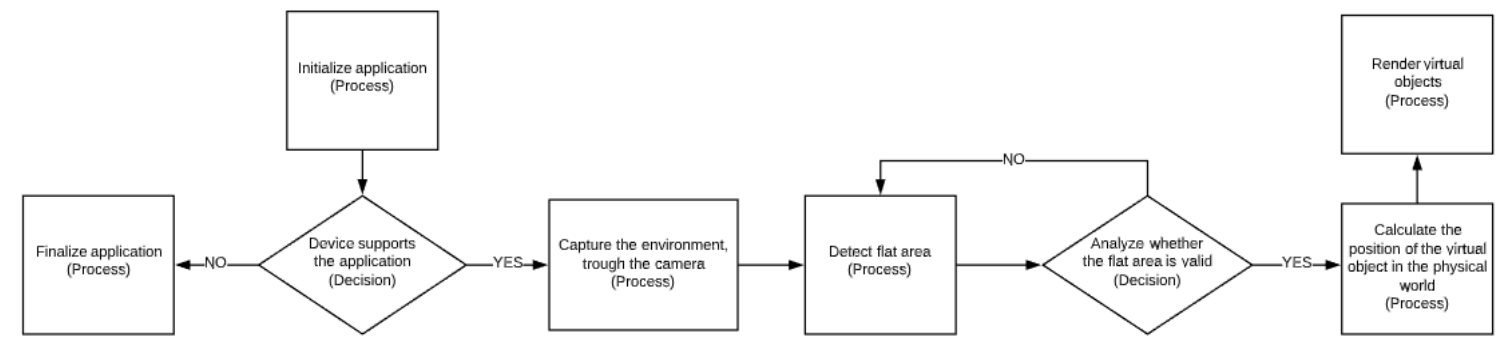

Figure 3. Flow chart of the functioning of augmented reality in BNAnatomy

The prototype was subjected to tests that allowed us to reach conclusions about aspects of its usability. Six participants, male and female, all students or professionals in health-related areas, answered a total of 15 questions, all of them subjective, divided into two questionnaires. They answered the questions with values from 1 to 5 , with grade 1 being defined as the lowest level of approval and grade 5 as the highest level of approval possible. 
The first questionnaire included a total of 9 questions, all related to aspects of system interaction and interface. The systematic review by Martins et al. (2015) served as a starting point for formulating these questions. These are the questions from the first questionnaire: q0) is the object easy to handle? ; q1) is gestural control compatible with movement? ; q2) was the text provided in this system clear? ; 3 ) was the audio feedback clear?; q4) is the application response / latency time appropriate? ; q5) is it possible to notice that the objects are in 3D? ; q6) is it difficult to make mistakes in the application? ; q7) does the application report your progress? ; q8) is the application easy to start? .

The second questionnaire had 6 questions regarding the learning aspects. The following questions were covered in this: q0) were you satisfied with the application? ; q1) do you believe that the application is relevant to the field of knowledge? ; q2) did you find the application fun? ; q3) does the application provide a learning facility superior to conventional teaching methodologies? ; q4) does application bring a greater motivation for learning than conventional teaching methodologies? ; q5) would you recommend it to a friend (student or professional) in the health field? .

\section{RESULTS AND DISCUSSION}

\subsection{Results of the Questionnaire about the System and Interface}

The results show that the system is easy to be manipulated, intuitive, with clear commands and with acceptable latency, but users have shown a lack of information about their progress. Table 1 shows the average of user ratings for the questions in the first questionnaire.

Table 1. Average of the evaluations obtained on the questions related to the first questionnaire

\begin{tabular}{cc}
\hline Question & Average \\
\hline Q0) Is the object easy to handle? & 4.17 \\
Q1) Is gestural control compatible with movement? & 4.83 \\
Q2) Was the text provided in this system clear? & 4.50 \\
Q3) Was the audio feedback clear? & 4.83 \\
Q4) Is the application response / latency time appropriate ? & 4.00 \\
Q5) Is it possible to notice that the objects are in 3D? & 5.00 \\
Q6) Is it difficult to make mistakes in the application? & 4.00 \\
Q7) Does the application report your progress? & 3.67 \\
Q8) Is the application easy to start? & 4.83 \\
\hline
\end{tabular}

\subsection{Results of the Questionnaire on the Learning Aspects}

Regarding the learning aspects, the answers were very satisfactory, showing a great degree of enthusiasm from the volunteers with the approach that we propose for teaching bone anatomy. Table 2 shows the average user ratings for the questions in the second questionnaire.

Table 2. Average of the evaluations obtained on the questions related to the second questionnaire

\begin{tabular}{cc}
\hline Question & Average \\
\hline Q0) Were you satisfied with the application? & 4.83 \\
Q1) Do you believe that the application is relevant to the field of knowledge? & 5.00 \\
& 4.67 \\
Q3)Does the application provide a learning facility superior to conventional teaching methodologies? & 5.00 \\
Q4) Does application bring a greater motivation for learning than conventional teaching methodologies? & 5.00 \\
Q5) Would you recommend it to a friend (student or professional) in the health field? & 4.83 \\
\hline
\end{tabular}




\section{CONCLUSION}

Despite the research being in its initial state, the results obtained with the tests were encouraging. We found evidence of a considerable relevance of the solution presented in this paper for the study of bone anatomy.

Regarding aspects that involve learning, the evaluations collected reveal a satisfaction with the application by the volunteers. In addition, almost all users claim that the experience stands out from conventional teaching methodologies, such as textbooks and atlas of human anatomy. We believe that this results from both the augmented reality experience and the proposed gamification.

The opinions about the interface and system were also positive; all users were able to use the application intuitively, without monitoring or guidance from third parties. However, part of the volunteers reported not being able to accurately understand their progress within the proposed experience in the system.

Although the application limits its operation only to cell phones with the Android operating system above version 7.0, it remains more accessible and cheaper than other similar solutions. Finally, we believe in the potential of the solution presented to add value to the teaching of bone anatomy. However, we see the need to add more bone models to the experiment and the reproduction of the experiment in a larger sample.

\section{ACKNOWLEDGMENT}

The authors are thankful to the volunteers that answered the questionnaires. A. C. thanks to INCT-MACC and CNPq, Universal 402988/2016-7 and PQ 305416/2018-9 Projects.

\section{REFERENCES}

Andayani, U., Siregar, B., Hernina Pulungan, S., Syahputra, M.F., Muchtar, M.A., Arisandi, D., 2019. A Visualisation of 3D Lung Anatomy with Augmented Reality as Interactive Medical Learning. J. Phys.: Conf. Ser. 1235, 012095. https://doi.org/10.1088/1742-6596/1235/1/012095

Azuma, R., Baillot, Y., Behringer, R., Feiner, S., Julier, S., MacIntyre, B., 2001. Recent advances in augmented reality. IEEE Comput. Grap. Appl. 21, 34-47. https://doi.org/10.1109/38.963459

Ferrer-Torregrosa, J., Jiménez-Rodríguez, M.Á., Torralba-Estelles, J., Garzón-Farinós, F., Pérez-Bermejo, M., Fernández-Ehrling, N., 2016. Distance learning ects and flipped classroom in the anatomy learning: comparative study of the use of augmented reality, video and notes. BMC Med Educ 16, 230. https://doi.org/10.1186/s12909-016-0757-3

Kuhn, C.M., Flanagan, E.M., 2017. Self-care as a professional imperative: physician burnout, depression, and suicide. Can J Anesth/J Can Anesth 64, 158-168. https://doi.org/10.1007/s12630-016-0781-0

Kurniawan, M.H., Suharjito, Diana, Witjaksono, G., 2018. Human Anatomy Learning Systems Using Augmented Reality on Mobile Application. Procedia Computer Science 135, 80-88. https://doi.org/10.1016/j.procs.2018.08.152

Martins, V.F., 2012. Desafios para o uso de Realidade Virtual e Aumentada de maneira efetiva no ensino 10.

Martins, V.F., Kirner, T.G., Kirner, C., 2015. Subjective Usability Evaluation Criteria of Augmented Reality Applications, in: Shumaker, R., Lackey, S. (Eds.), Virtual, Augmented and Mixed Reality, Lecture Notes in Computer Science. Springer International Publishing, Cham, pp. 39-48. https://doi.org/10.1007/978-3-319-21067-4_5

Singh, M., Singh, M.P., 2013. Augmented Reality Interfaces. IEEE Internet Comput. 17, 66-70. https://doi.org/10.1109/MIC.2013.107

Stefan, P., Wucherer, P., Oyamada, Y., Ma, M., Schoch, A., Kanegae, M., Shimizu, N., Kodera, T., Cahier, S., Weigl, M., Sugimoto, M., Fallavollita, P., Saito, H., Navab, N., 2014. An AR edutainment system supporting bone anatomy learning, in: 2014 IEEE Virtual Reality (VR). Presented at the 2014 IEEE Virtual Reality (VR), IEEE, Minneapolis, MN, USA, pp. 113-114. https://doi.org/10.1109/VR.2014.6802077 